\title{
On bias of kinetic temperature measurements in complex plasmas
}

\author{
Kantor, M.; Moseev, D.; Salewski, Mirko
}

Published in:

Physics of Plasmas

Link to article, DOI:

$10.1063 / 1.4864326$

Publication date:

2014

Document Version

Publisher's PDF, also known as Version of record

Link back to DTU Orbit

Citation (APA):

Kantor, M., Moseev, D., \& Salewski, M. (2014). On bias of kinetic temperature measurements in complex plasmas. Physics of Plasmas, 21(2), 023701. https://doi.org/10.1063/1.4864326

\section{General rights}

Copyright and moral rights for the publications made accessible in the public portal are retained by the authors and/or other copyright owners and it is a condition of accessing publications that users recognise and abide by the legal requirements associated with these rights.

- Users may download and print one copy of any publication from the public portal for the purpose of private study or research.

- You may not further distribute the material or use it for any profit-making activity or commercial gain

- You may freely distribute the URL identifying the publication in the public portal

If you believe that this document breaches copyright please contact us providing details, and we will remove access to the work immediately and investigate your claim 


\title{
On bias of kinetic temperature measurements in complex plasmas
}

\author{
M. Kantor, ${ }^{1,2,3}$ D. Moseev, ${ }^{1,2, a)}$ and M. Salewski ${ }^{4}$ \\ ${ }^{1}$ Association Euratom-Max-Planck-Institut für Plasmaphysik, D-85748 Garching bei München, Germany \\ ${ }^{2}$ Association Euratom-FOM Institute DIFFER, 3430 BE Nieuwegein, The Netherlands \\ ${ }^{3}$ Ioffe Institute, RAS, St. Petersburg 194021, Russia \\ ${ }^{4}$ Association Euratom-DTU, Department of Physics, Technical University of Denmark, DTU Ris o Campus, \\ DK-4000 Roskilde, Denmark
}

(Received 4 December 2013; accepted 15 January 2014; published online 7 February 2014)

\begin{abstract}
The kinetic temperature in complex plasmas is often measured using particle tracking velocimetry. Here, we introduce a criterion which minimizes the probability of faulty tracking of particles with normally distributed random displacements in consecutive frames. Faulty particle tracking results in a measurement bias of the deduced velocity distribution function and hence the deduced kinetic temperature. For particles with a normal velocity distribution function, mistracking biases the obtained velocity distribution function towards small velocities at the expense of large velocities, i.e., the inferred velocity distribution is more peaked and its tail is less pronounced. The kinetic temperature is therefore systematically underestimated in measurements. We give a prescription to mitigate this type of error. [http://dx.doi.org/10.1063/1.4864326]
\end{abstract}

\section{INTRODUCTION}

Dusty particles in complex plasmas are usually described by two different temperatures: the surface temperature of the dust particles and the kinetic temperature which describes the chaotic motion of the dust particles. Accurate knowledge of the kinetic temperature is essential for heattransfer studies in complex plasmas. ${ }^{1-3}$ Complex plasma is also used to study phase transition in crystalline structures ${ }^{3-5}$ where correct measurements of the kinetic temperature are important. Usually, the kinetic temperature is measured by means of high-speed cameras which record projections of dust particle locations on the image planes of the cameras. Then 2D projections of the trajectories can be inferred from these images. If two or more cameras are used, 3D trajectories can be reconstructed. ${ }^{6-10}$ This method is called particle tracking velocimetry (PTV). It is used in fluid dynamics to study flows, ${ }^{8,10-14}$ in combustion physics, ${ }^{15,16}$ in fusion research, ${ }^{17-19}$ and in complex plasma physics. ${ }^{9,20-22}$

There are several types of errors which may occur during PTV measurements, for example, errors related to particle acceleration, ${ }^{23,24}$ uncertainties in particle positions due to finite camera resolution, ${ }^{23,25}$ or wrongly reconstructed particle locations as a result of measurement ambiguities. ${ }^{10}$ In this paper, we study the problem of assigning trajectories to particles, i.e., finding correspondence between indistinguishable particles in consecutive frames which is also a source of errors. Velocity distribution function measurements require correct tracking of the particles leading to correct trajectory assignments. However, we will show that incorrect tracking of particles will lead to deformation of the velocity distribution function and hence to significant errors in kinetic temperature measurements as well as measurements of other quantities that depend on the velocity distribution function. If the particles were distinguishable, any given particle could

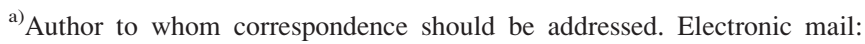
dmitry.moseev@ipp.mpg.de
}

be uniquely identified in the next frame, and hence all particles in one frame could be matched to a particle in the next frame. However, if the particles are indistinguishable, it is not possible to identify a given particle in one frame uniquely in the next frame. Hence, mismatches of particles are bound to occur. This will lead faulty trajectory and velocity assignments of the mismatched particles. Therefore, the velocity distribution function will be distorted. We will show that the measured velocity distribution function will be biased towards smaller velocities at the expense of larger velocities, leading to a bias towards lower kinetic temperatures.

The results demonstrated in this paper are applicable not only to complex plasmas but also to colloids ${ }^{26-29}$ and granular materials. ${ }^{30-34}$

In Sec. II, we formulate the problem and introduce a matching criterion as the basis for its solution which we present in Sec. III. In Sec. IV, we derive analytical expressions for the probabilities of correct tracking of two particles. In Sec. V, we derive analytical expressions for the biased distribution of their random displacements caused by faulty tracking. These results are tested numerically in Sec. VI, where we study the probability of mistracking and biasing the distribution function for a large number of particles. Section VII concludes the paper.

\section{PROBLEM FORMULATION AND MATCHING CRITERION}

Let us consider a group of indistinguishable particles in a volume viewed by several cameras. The cameras are synchronized and take a series of $2 \mathrm{D}$ images of projections of particle positions on their image planes. For simplicity, we assume here that the particle positions in 3D can be unambiguously reconstructed from a set of simultaneously acquired 2D camera images which we refer to as frame. To deduce the particle trajectories, particles in consecutive frames have to be matched. If they were distinguishable, this 
would be easy. But since they are indistinguishable, it is impossible to identify any given particle in the next frame. The problem of particle tracking is formulated as a search of 3D trajectories of individually moving particles from a sequence of frames. We assume that no particles leave or enter the observation volume. Particle trajectories can be deduced from coordinates in consecutive frames. In the case of regular motion, one can predict the coordinates of the particles in the next frame from their deduced velocities in previous frames and aid the correct matching of particles in consecutive frames. The tracking problem arises when the particles randomly deviate from their predicted trajectories. A possible tracking error can be especially significant when the random deviation is comparable with or larger than the inter-particle distance. In this paper we give a particle matching criterion to ensure the statistically optimal tracking of chaotically moving particles for any inter-particle distance. We derive expressions for the bias of statistical parameters of measured trajectories which deviate from the real trajectories.

Further analysis deals with the chaotic component of the particle motion, whereas we subtract out the regular component of the motion. The regular component can be found by extrapolation of particle coordinates from their coordinates in previous frames and their instantaneous velocities in the previous frame. Random differences between extrapolated particle positions and actual particle positions are referred to as jumps between frames. These jumps make up the chaotic component of the particle motion.

\section{MATCHING CRITERION}

When jumps are much smaller than inter-particle distances, a particle trajectory can be efficiently reconstructed just by choosing the particle that is closest to its predicted position. However, if the particle jump size is comparable to the inter-particle distance, such a simple assignment is not possible. For jumps of arbitrary length, in particular, large jumps comparable to the inter-particle distance, we formulate the matching condition as a minimization problem as follows. We consider a normal isotropic distribution of particle jumps

$$
f(s)=\frac{1}{\Delta \sqrt{2 \pi}} \exp \left(-\frac{s^{2}}{2 \Delta^{2}}\right),
$$

where $s$ is the projected displacement from the predicted particle position. $\Delta^{2}$ is its variance and scales inversely with the frame rate of detection cameras.

$N$ particles in two consecutive frames can be matched in $N$ ! possible ways. The probability density of one particular way is the product of the probability densities of jumps for all $N$ particles. Hence, the probability density $P_{k}$ of a particular way $k$ becomes

$$
P_{k}=\left(\frac{1}{\Delta \sqrt{2 \pi}}\right)^{N} \exp \left(-\frac{\sum_{i=1}^{N} \sum_{j=x, y, z} s_{i j k}^{2}}{2 \Delta^{2}}\right) .
$$

The realization with maximum probability density is the one with least squared sum of jumps, and hence the matching criterion becomes:

$$
\text { minimize: } S_{k}^{2}=\sum_{i=1}^{N} \sum_{j=x, y, z} s_{i j k}^{2} \text {, }
$$

where $S_{k}^{2}$ stands for the sum of squared jump lengths of all particles from one frame to the $k^{\text {th }}$ of $N$ ! possible permutations of particles in the successive frame.

We choose a normal distribution of jumps due to its simplicity for further analysis and universality. However, in many experiments with abnormal diffusion, deviations from the Gaussian pdfs were reported. ${ }^{35-38}$ The matching criterion for the non-Gaussian distribution of jumps can be formulated in a similar way by maximizing the probability density $P_{k}$ of a particular way $k$.

\section{PROBABILITY OF PARTICLE MISTRACKING}

The matching criterion in Eq. (3) provides the statistically best way of tracking the particles recorded in two consecutive frames. However, this does not imply that all particles are tracked correctly. We illustrate and quantify a systematic tracking error in the case of just two particles in the following. Consider the situation depicted in Figure 1. The filled red and the empty blue circles denote two particles at two instances. Continuous arrows show true jumps of the particles of the same color. However, according to the matching criterion, the trajectory reconstruction algorithm would assign the filled red particle as empty blue and the empty blue particle as filled red in the next time step. Dashed lines indicate faulty trajectories which appear as a result of such a switching event.

Now we quantify the probability of this event. Let the particles be distributed uniformly in space and jumps in two consecutive frames be distributed normally (Sec. III). Suppose that the distance between the particles is $r$. Now, we construct a new Cartesian coordinate system so that its origin is located halfway between the particles. Particle 1 has coordinates $(-r / 2,0,0)$ and particle 2 has coordinates $(r / 2,0,0)$. The probabilities for particle 1 to change its current position to the range between $\left(x_{1}, x_{1}+d x_{1}\right)$ and for particle 2 to

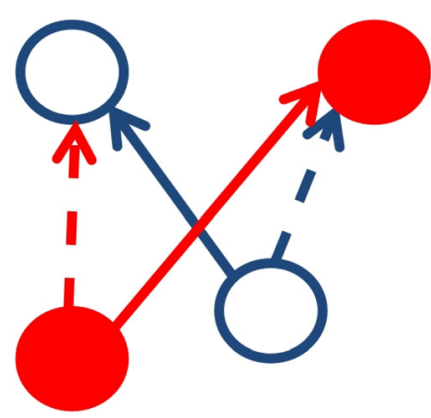

FIG. 1. Demonstration of faulty particle matching leading to faulty trajectories for both particles. Filled red and empty blue circles represent two particles in two consecutive frames at times $t$ and $t+\delta t$. Continuous arrows denote true directions of movement. Dashed arrows represent faulty trajectories that are assigned by the algorithm. 
change its current position to the range $\left(x_{2}, x_{2}+d x_{2}\right)$ are given by

$$
\begin{aligned}
& d P_{1}=\frac{d x_{1}}{\Delta \sqrt{2 \pi}} \exp \left(-\frac{\left(x_{1}+r / 2\right)^{2}}{2 \Delta^{2}}\right) \\
& d P_{2}=\frac{d x_{2}}{\Delta \sqrt{2 \pi}} \exp \left(-\frac{\left(x_{2}-r / 2\right)^{2}}{2 \Delta^{2}}\right) .
\end{aligned}
$$

The particles are tracked incorrectly if $x_{1}>x_{2}$ as we illustrate in Figure 2. The probability $P_{12 i}$ of incorrect tracking of two particles separated by distance $r$ is given by the integration of the product of $d P_{1}$ and $d P_{2}$ in the region $x_{1}>x_{2}$, as it is given in the following equation:

$$
\begin{aligned}
P_{12 i} & =\frac{1}{2 \pi \Delta^{2}} \int_{-\infty}^{\infty} d x_{1} \int_{-\infty}^{x_{1}} d x_{2} \exp \left(-\frac{\left(x_{1}+r / 2\right)^{2}+\left(x_{2}-r / 2\right)^{2}}{2 \Delta^{2}}\right) \\
& =0.5\left(1-\operatorname{erf}\left(\frac{r}{2 \Delta}\right)\right)
\end{aligned}
$$

where erf stands for the error-function. The integration is done using Ref. 39. The probability of correct tracking is

$$
P_{12 c}=1-P_{12 i}=0.5+0.5 \operatorname{erf}\left(\frac{r}{2 \Delta}\right) .
$$

For $\Delta \gg r, P_{12 c}$ converges to 0.5 , corresponding to the $50 \%$ chance to match 2 particles correctly if the jump size is much larger than the inter-particle distance.

We derived the probability of incorrect tracking according to Eq. (5) for particles separated by a fixed distance $r$. However, a random spatial distribution of particles results in random distances between them. If the distribution of distances between neighbouring particles is a random variable described by the distribution function $f_{2}$, the incremental probability $d P_{i}$ of faulty tracking becomes $d P_{i}=P_{12 i} f_{2} d r$. From here on, we assume that particles are distributed uniformly in space in the first frame. Hence, according to Ref. 40, the distribution of distances between neighbouring particles is

$$
f_{2}(r, \lambda)=4 \pi \lambda r^{2} \exp \left(-\frac{4 \pi \lambda r^{3}}{3}\right),
$$

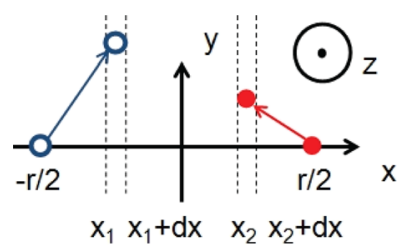

(a)

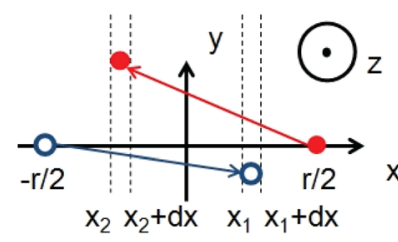

(b)
FIG. 2. Schematic of a new coordinate system and particles displacements from one frame to another. Particle 1 (empty blue circles) moves in between frames to a new position between two planes $x=x_{1}$ and $x=x_{1}+d x$. The displacement is schematically depicted by the blue arrow. Particle 2 (filled red circles) moves to a new position between two planes $x=x_{2}$ and $x=x_{2}+$ $d x$. The displacement is schematically depicted by the red arrow. In (a) $x_{1}<x_{2}$, reconstructed trajectories of two particles coincide with the true trajectories; (b) $x_{1}>x_{2}$, particles 1 and 2 switch their positions in the reconstruction leading to faulty trajectory assignments. where $\lambda$ is the intensity of a $3 \mathrm{D}$ Poisson process. $\lambda$ relates to the mean squared distance between neighbouring particles in the following way:

$$
\left\langle r^{2}\right\rangle=\left(\frac{3}{4 \pi \lambda}\right)^{2 / 3} \Gamma(5 / 3),
$$

where $\Gamma$ denotes the Gamma-function.

The probability of correct tracking is then given by

$$
\begin{aligned}
P_{c} & =1-\int_{0}^{\infty} P_{12 i} f_{2} d x \\
& =\frac{1}{\sqrt{\pi}} \int_{0}^{\infty} \exp \left(-x^{2}\right)\left[1+\exp \left(-\frac{32 \pi \lambda \Delta^{3} x^{3}}{3}\right)\right] d x .
\end{aligned}
$$

Figure 3 shows a probability of correct tracking for random distances of uniformly distributed particles $P_{c}$ as function of normalized particle jump. The normalization is done to the root mean square distance between neighboring particles, which relates to the intensity of the $3 \mathrm{D}$ Poisson process $\lambda$ according to Eq. (8). The normalized jump $s_{N}$ in this case is defined in the following equation:

$$
s_{N}=\frac{\Delta}{\sqrt{\left\langle r^{2}\right\rangle}}
$$

Black circles in Figure 3 denote the result of a straightforward numerical simulation of the matching criterion according to Eq. (3). We find very good agreement between the predicted result according to theory and the numerical simulation.

\section{EFFECT OF MISTRACKING ON MEASURED VELOCITY DISTRIBUTION FUNCTIONS}

Application of the matching criterion results in peaking of tracked random velocities around zero. The predicted

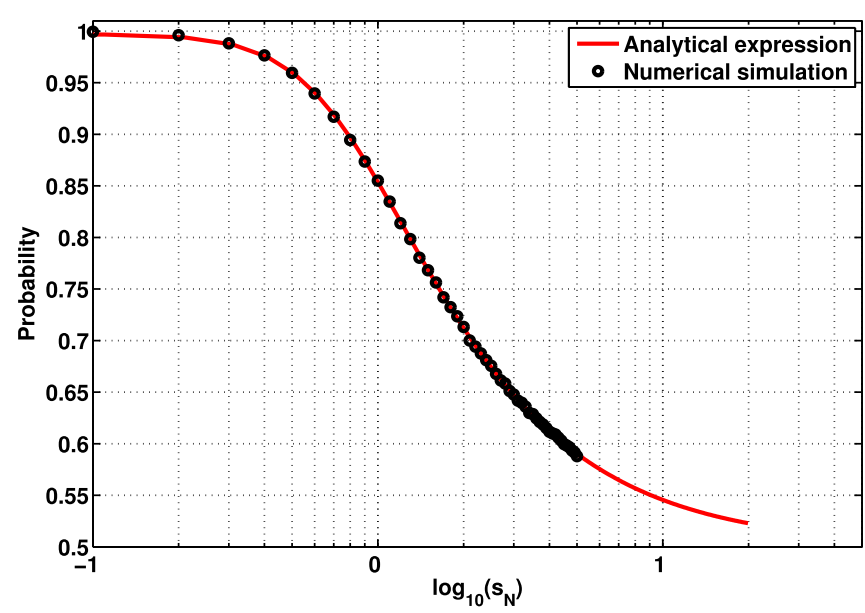

FIG. 3. Probability of correct tracking calculated for particles randomly and uniformly distributed in space (red solid line) plotted as a function of the normalized particle jump. Circles denote the result of a numerical simulation which will be discussed in Sec. VI. 
effect would be that the measured distribution function is more peaked and has a smaller variance. In the following, we confirm this point quantitatively. We would like to know the probability density of a particle to jump from one frame to another by a distance $\rho$. The distance to its nearest neighbour is $r$. Consider a spherical coordinate system with the origin at the particle of interest. The probability density function (pdf) to find the particle at distance $\rho$ from the origin consists of two terms. The first term describes the pdf that the particle really made a jump $\rho$ and we accounted for it correctly. The second term shows the pdf that the particle of interest jumped elsewhere and its nearest neighbour jumped such that we have mistaken it for the particle of interest at distance $\rho$ from its previous location:

$$
\begin{gathered}
\frac{\partial^{3} F(r, x, y, z, \Delta)}{\partial x \partial y \partial z}=f(x) f(y) f(z) \int_{z}^{\infty} f\left(z^{\prime}-r\right) d z^{\prime} \\
+f(x) f(y) f(z-r) \int_{z}^{\infty} f\left(z^{\prime}\right) d z^{\prime}, \\
x=\rho \cos (\phi) \sin (\theta), \quad y=\rho \sin (\phi) \sin (\theta), \\
z=\rho \cos (\theta), \quad \frac{\partial^{3} F(r, x, y, z, \Delta)}{\partial x \partial y \partial z} \rightarrow \frac{\partial^{3} F(r, \rho, \theta, \phi, \Delta)}{\rho^{2} \partial \rho \partial \Omega}, \\
\partial \Omega=\sin (\theta) \partial \theta \partial \phi .
\end{gathered}
$$

Here, $f$ stands for the normal distribution function as in equation (1), $r$ is the distance between particles in the first frame, the $z$-axis goes through two particles in the first frame, and $\theta$ is the angle between the $z$-axis and the candidate particle in the next frame.

The posed problem is completely isotropic in terms of $\phi$ and $\theta$ angles. Integration over them reduces the dimensionality of Eq. (11). The analytic expression for this integral is shown in the Appendix.

$$
\frac{\partial F(r, \rho, \Delta)}{\partial \rho}=\int_{0}^{2 \pi} d \phi \int_{0}^{\pi} d \theta \frac{\partial^{3} F(r, \rho, \theta, \phi, \Delta)}{\partial \rho \partial \Omega} \sin (\theta) .
$$

Integration of Eq. (12) over angle $\theta$ and averaging over $\rho$, which is distributed according to Eq. (7), gives a measurable distribution of radial jumps

$$
\frac{\partial f(\Delta, \lambda)}{\partial \rho}=\frac{1}{4 \pi} \int_{0}^{\infty} \frac{\partial F(r, \rho, \Delta)}{\partial \rho} f_{2}(r, \lambda) d r .
$$

In this equation, $4 \pi$ is already taken into account in Eq. (7) for $f_{2}$. Therefore, the value of the integral is divided by $4 \pi$ in order to avoid accounting for it twice. However, the measured quantity is the pdf of a projection of an isotropic radial particle jump on one Cartesian axis, according to the geometry of the considered problem

$$
\frac{\partial F(\Delta, \lambda)}{\partial s}=2 \pi \int_{s}^{\infty} \frac{\partial f(\Delta, \lambda)}{\partial \rho} \frac{d \rho}{\rho} .
$$

In the following, we choose a distribution function which we refer to as true. We sample particle jumps from this distribution function and follow them by particle tracking. We then simulate a PTV measurement from the known true particle trajectories. The measured distribution function is then different from the true distribution function due to the bias originating from tracking errors. The deduced distribution function is also plotted in Figure 4. The plot shows good agreement between the analytically obtained pdf and the pdf measured in the numerical experiment, where two particles are tracked for $10^{5}$ frames. The calculations are done for normalized jump $s_{N}=2$.

We fit the measured distribution with a Maxwellian function of the form

$$
f_{f i t}=A \cdot \exp \left(-\frac{s^{2}}{2 \Delta_{f i t}^{2}}\right)
$$

For comparison, we introduce a quantity $w$ which is the ratio of squared widths of the fitted Maxwellian and the true distribution function. It can be interpreted as the ratio of the measured and true kinetic temperatures, if we define the kinetic temperature as squared width of the fitted Gaussian

$$
w=\left(\frac{\Delta_{f i t}}{\Delta}\right)^{2}
$$

We also compare actual second moments of measured and true distribution functions. This can also be understood as the ratio of the measured and true kinetic temperatures, if we define the kinetic temperature as the second moment of the measured velocity distribution function

$$
h=\frac{M 2\left(f_{m}\right)}{\Delta^{2}},
$$

where $M 2\left(f_{m}\right)$ denotes the second moment of the measured distribution function. We also calculate the deviation of the measured distribution function from normal by plotting its

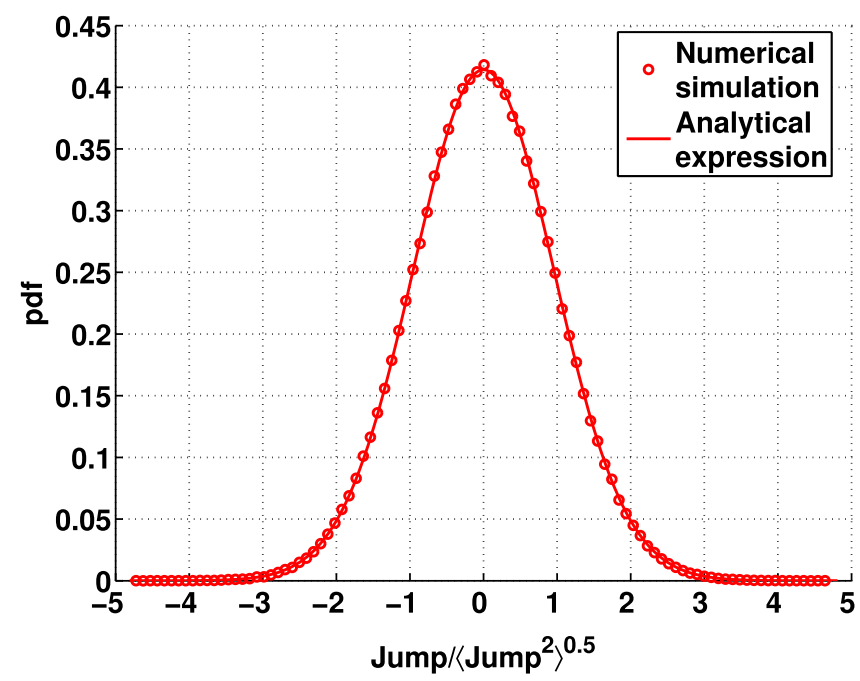

FIG. 4. Measured (circles) and analytically calculated (solid line) pdfs of particle displacements. The graph is plotted for two particles tracked over $10^{5}$ frames and normalized jump $s_{N}=2$. 
reduced moment $m$, which is always equal to zero for the normal distribution

$$
m=\frac{M 4\left(f_{m}\right)}{M 2\left(f_{m}\right)^{2}}-3,
$$

where $M 4\left(f_{m}\right)$ denotes the fourth moment of the measured distribution function. The results of the comparison are shown in Figure 5 as a function of dimensionless parameter $s_{N}$. Deviations of the reduced moment $m$ from zero show that the measured displacement distribution function is not Maxwellian, although it has similar appearance and can be well fitted by a single normal distribution, but with different width.

In Figure 5, we also compare our theoretical model with numerical simulations in which two particles were tracked for $10^{5}$ frames. The very good agreement between theory and the numerical simulation demonstrates that the nature of the measurement-induced systematic error due to faulty particle matching is well understood. The results for the two-particle model can also be used when more particles are present in the frame, if the fraction of swapping trajectories of three or more particles is negligible. The case of three or more particles is addressed quantitatively in Sec. VI.

\section{NUMERICAL TRACKING}

Analytical results from Sec. IV are obtained for two particles. Usually more than two particles are tracked. Nevertheless, the two-particle case gives useful insight into the mechanisms of particle mistracking. Here, we extend our results to more than two particles by straightforward numerical implementation of the matching criterion that we used
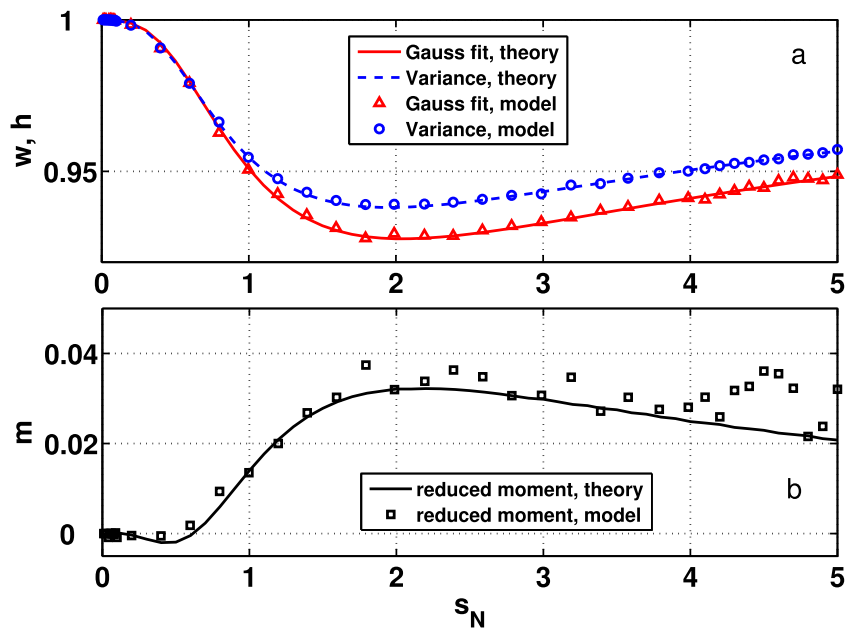

FIG. 5. Analysis of tracking in two particle system as a function of normalized displacement $s_{N}$. (a) Ratio between measured and true second moments of the distribution function $h$ (blue dashed line-analytical solution, blue circles-numerical experiment); ratio of squared widths of the fitted Gaussian to the measured distribution function and variance of the true distribution function $w$ (red solid line-analytical solution, red triangles-numerical experiment); (b) reduced moment $m$ of the measured distribution function of displacements (black solid line-analytical solution, black circles-numerical experiment). The numerical experiment is performed for $10^{6}$ realizations. for the analytical analysis of the probability of correct tracking. We again assume a uniform distribution of particles in space and random isotropic jumps between frames.

For two particles in a frame, the maximum possible error in the kinetic temperature measurements is below $10 \%$. However, when many particles per frame have to be tracked, large errors can occur. Figure 6 shows an example of a true (red dashed line) and a measured (blue solid line) distribution function where nine particles are tracked over $10^{6}$ frames with a normalized jump size of $s_{N}=2$. The ratio of variances of the measured and true jump distributions is $74 \%$. The contour plots depicted in Figure 7 show the probability of correct tracking (a), the ratio of squared widths of the fitted Gaussian and the true distribution function (b), the ratio of second moments of the measured and true velocity distribution functions (c), and the reduced moment of the measured distribution function (d) as functions of the number of particles and their normalized jumps. One can see that for relatively small normalized particle jumps, the probability of correct tracking, as well as temperature ratios are almost independent of the number of tracked particles. This observation quantifies the statement made in Sec. V that for relatively small normalized jumps the probability of particle mistracking and the shape of the measured distribution function are described analytically by the two-particle model introduced in Secs. IV and V. The form of this plot allows us to choose a working point (i.e., lowest possible framing rate) of the instrument based on expected temperature estimations and a maximum tolerable probability of incorrect tracking.

Reduced moments, depicted in Figure 7(d), show how the measured distribution function deviates from the normal distribution for which the reduced moment $m$ is always zero. For small normalized jumps, the normal distribution is a good approximation for the measured distribution function. For larger number of particles in the frame, the normal distribution is a good approximation for larger values of the normalized jumps. However, this trend saturates when $s_{N}=1$. For larger $s_{N}$, the measured distribution functions deviates from the Gaussian. The larger $s_{N}$, the larger the deviation becomes.

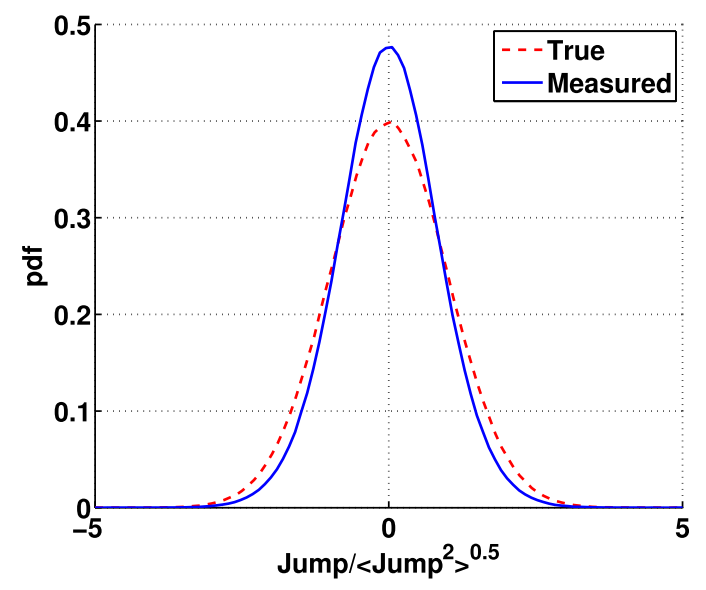

FIG. 6. Measured (blue solid line) and true (red dashed line) distributions of particle jumps, obtained for nine particles per frame and tracked over $10^{6}$ frames. Normalized jump $s_{N}=2$. Ratio of variances $h=0.74$. 


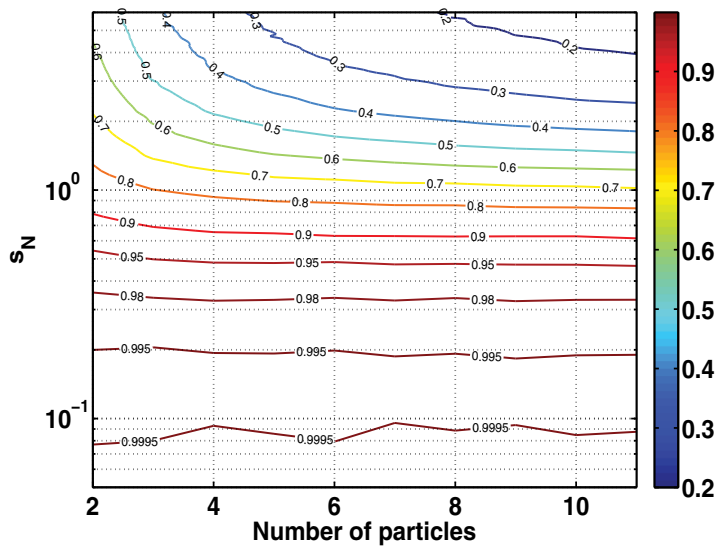

(a)

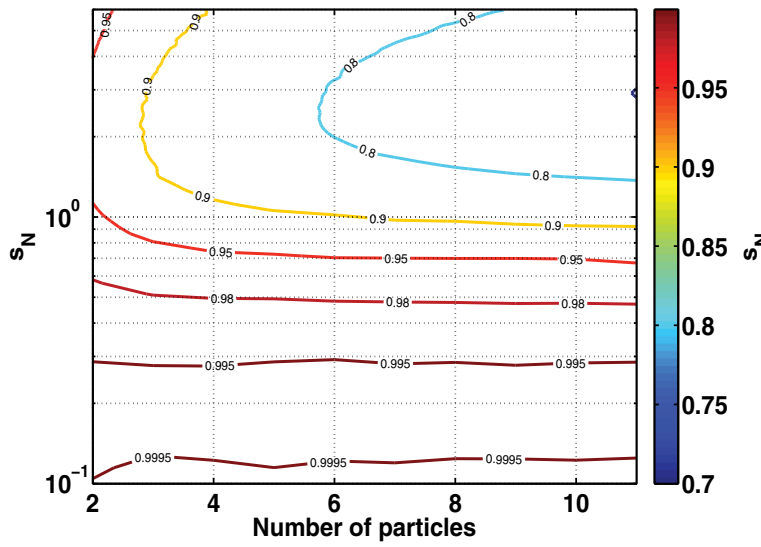

(c)

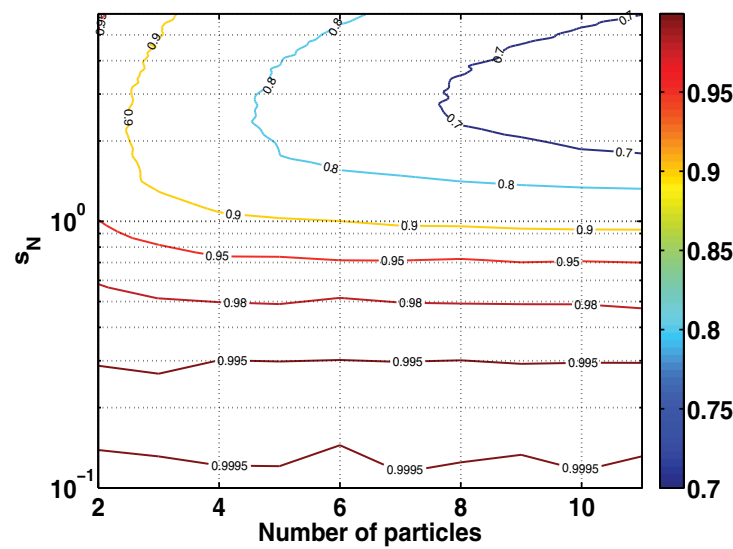

(b)

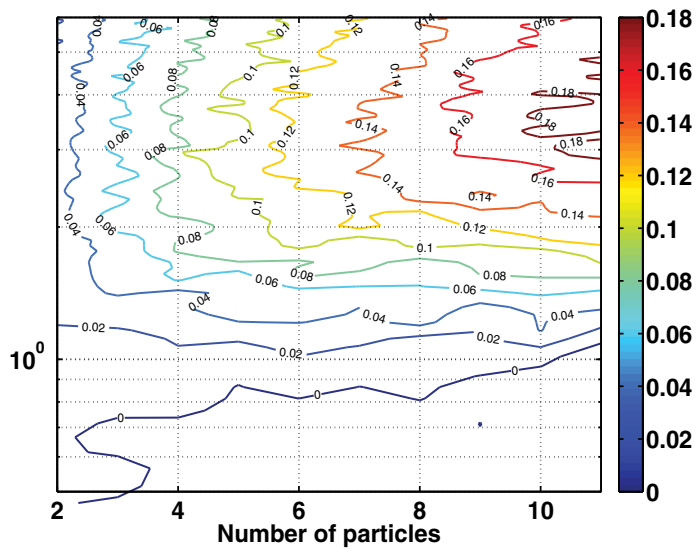

(d)

FIG. 7. Contour plots which show the probability of correct particle tracking (a); ratio of squared widths $w$ of the fitted Gaussian to the measured distribution function and the true distribution function (b); ratio $h$ of second moments of the measured and true distribution function (c); reduced moment $m$ of the measured distribution function (d).

\section{CONCLUSIONS}

We have derived a matching criterion which provides the highest fidelity of particle tracking when the chaotic component of particle velocities is normally distributed. However, faulty tracking is bound to occur. We have derived analytic expressions for the probability of correct tracking for two particles and arbitrary jump size. The expressions are also valid for more than two particles assuming that the average particle jump size between frames is smaller than the root mean square distance between neighbouring particles. From this, we have found the effect of mistracking on the deduced distribution function. Mistracking biases the deduced velocity distribution function towards smaller velocities. This bias has up to now not been considered.

We showed numerically that for small normalized jumps mistracking between two particles is a dominant mechanism of deformation of the measured velocity distribution function. This allows choosing a minimum tolerable framing rate for cameras, which is important because the error due to finite camera resolution is proportional to the framing rate. ${ }^{23}$

Generally, the measured distribution function is not Gaussian, although for small normalized jumps it is a good approximation. However, one has to remember that for tracking a large number of particles with non-exact algorithms (those which do not use a straightforward implementation of the matching criterion), the measured distribution function can be strongly non-Maxwellian and the difference in temperature definition (i.e., the second moment of the measured velocity distribution function or the squared width of the fitted Maxwellian distribution) can make a substantial difference.

\section{ACKNOWLEDGMENTS}

Dmitry Moseev was supported by the EFDA fellowship. This work, supported by the European Communities under the Contract of Association between EURATOM, DTU, FOM Institute DIFFER, and IPP, was carried out within the framework of the European Fusion Programme. The views and opinions expressed herein do not necessarily reflect those of the European Commission.

\section{APPENDIX: ANALYTICAL EXPRESSION}

The probability density to detect displacement $\rho$ of a particle, which is separated by distance $r$ from its neighbour, is determined by the following equation: 


$$
\begin{aligned}
\frac{\partial F(r, \rho, \Delta)}{\partial \rho}= & \frac{\rho^{2}}{\sqrt{2 \pi} \Delta^{3}} \exp \left(-\frac{\rho^{2}}{2 \Delta^{2}}\right)+\frac{\rho^{2} \exp \left(-\frac{\rho^{2}}{2 \Delta^{2}}\right)}{\sqrt{2 \pi} \Delta^{3}} \cdot \frac{\operatorname{erf}\left(\frac{r+\rho}{\sqrt{2} \Delta}\right)+\operatorname{erf}\left(\frac{r-\rho}{\sqrt{2} \Delta}\right)}{2} \\
& +\frac{\rho r \exp \left(-\frac{\rho^{2}}{2 \Delta^{2}}\right)}{\sqrt{2 \pi} \Delta^{3}} \cdot \frac{\operatorname{erf}\left(\frac{r+\rho}{\sqrt{2} \Delta}\right)-\operatorname{erf}\left(\frac{r-\rho}{\sqrt{2} \Delta}\right)}{2}+\frac{\rho \exp \left(-\frac{\rho^{2}}{2 \Delta^{2}}\right)}{r \sqrt{2 \pi} \Delta} \cdot \frac{\operatorname{erf}\left(\frac{r+\rho}{\sqrt{2} \Delta}\right)-\operatorname{erf}\left(\frac{r-\rho}{\sqrt{2} \Delta}\right)}{2} \\
& +\frac{\rho}{r} \frac{\exp \left(-\frac{(\rho-r)^{2}}{2 \Delta^{2}}\right)-\exp \left(-\frac{(\rho+r)^{2}}{2 \Delta^{2}}\right)}{2 \sqrt{2 \pi} \Delta}-\frac{\rho}{r} \frac{\operatorname{erf}\left(\frac{\rho}{\sqrt{2} \Delta}\right)}{\sqrt{2 \pi} \Delta} \cdot \frac{\exp \left(-\frac{(\rho-r)^{2}}{2 \Delta^{2}}\right)+\exp \left(-\frac{(\rho+r)^{2}}{2 \Delta^{2}}\right)}{2} \\
& -\frac{\rho \exp \left(-\frac{\rho^{2}}{2 \Delta^{2}}\right)}{2 \pi \Delta^{2}}\left(\exp \left(-\frac{(\rho-r)^{2}}{2 \Delta^{2}}\right)-\exp \left(-\frac{(\rho+r)^{2}}{2 \Delta^{2}}\right)\right) .
\end{aligned}
$$

${ }^{1}$ V. Fortov, O. Vaulina, O. Petrov, M. Vasiliev, A. Gavrikov, I. Shakova, N. Vorona, Yu. Khrustalyov, A. Manohin, and A. Chernyshev, "Experimental study of the heat transport processes in dusty plasma fluid," Phys. Rev. E 75, 026403 (2007).

${ }^{2}$ Y. Feng, J. Goree, and B. Liu, "Solid superheating observed in twodimensional strongly coupled dusty plasma," Phys. Rev. Lett. 100, 205007 (2008).

${ }^{3}$ S. Nunomura, D. Samsonov, S. Zhdanov, and G. Morfill, "Heat transfer in a two-dimensional crystalline complex (dusty) plasma," Phys. Rev. Lett. 95, 025003 (2005).

${ }^{4}$ A. Melzer, A. Homann, and A. Piel, "Experimental investigation of the melting transition of the plasma crystal," Phys. Rev. E 53, 2757-2766 (1996).

${ }^{5}$ S. K. Zhdanov, M. H. Thoma, C. A. Knapek, and G. E. Morfill, "Compact dislocation clusters in a two-dimensional highly ordered complex plasma," New J. Phys. 14, 023030 (2012).

${ }^{6}$ A. Alpers, P. Gritzmann, D. Moseev, and M. Salewski, "3D particle tracking velocimetry using dynamic discrete tomography," pre-print arXiv:1307.4336 (2013).

${ }^{7}$ Y. Guezennec, R. Brodkey, N. Trigui, and J. Kent, "Algorithms for fully automated three-dimensional particle tracking velocimetry," Exp. Fluids 17, 209-219 (1994)

${ }^{8}$ F. Pereira, H. Stüer, E. C. Graff, and M. Gharib, "Two-frame 3D particle tracking," Measur. Sci. Technol. 17, 1680-1692 (2006).

${ }^{9}$ V. Hadziavdic, F. Melandso, and A. Hanssen, "Particle tracking from image sequences of complex plasma crystals," Phys. Plasmas 13, 053504 (2006).

${ }^{10} \mathrm{~B}$. Wieneke, "Iterative reconstruction of volumetric particle distribution," Measur. Sci. Technol. 24, 024008 (2013).

${ }^{11} \mathrm{~J}$. Katz and J. Sheng, "Applications of holography in fluid mechanics and particle dynamics,” Annu. Rev. Fluid Mech. 42, 531-555 (2010).

${ }^{12} \mathrm{R}$. Adrian, "Multi-point optical measurements of simultaneous vectors in unsteady flow-A review," Int. J. Heat Fluid Flow 7, 127-145 (1986).

${ }^{13}$ H. G. Maas, A. Gruen, and D. Papantoniou, "Particle tracking velocimetry in three-dimensional flows," Exp. Fluids 15, 133-146 (1993).

${ }^{14}$ R. Kieft, K. Schreel, G. van der Plas, and C. Rindt, "The application of a 3D PTV algorithm to a mixed convection flow," Exp. Fluids 33, 603-611 (2002).

${ }^{15}$ J. Pareja, H. Burbano, and Y. Ogami, "Measurements of the laminar burning velocity of hydrogen-air premixed flames," Int. J. Hydrogen Energy 35, 1812-1818 (2010)

${ }^{16} \mathrm{X}$. Qin, H. Kobayashi, and T. Niioka, "Laminar burning velocity of hydrogen-air premixed flames at elevated pressure," Exp. Therm. Fluid Sci. 21, 58-63 (2000).

${ }^{17}$ S. Rosanvallon, C. Grisolia, G. Counsell, S. Hong, F. Onofri, J. Worms, J. Winter, B. Annaratone, G. Maddaluno, and P. Gasior, "Dust control in tokamak environment," Fusion Eng. Des. 83, 1701-1705 (2008).

${ }^{18}$ S. I. Krasheninnikov, R. D. Smirnov, and D. L. Rudakov, "Dust in magnetic fusion devices," Plasma Physics Controlled Fusion 53, 083001 (2011).

${ }^{19}$ D. L. Rudakov, A. Litnovsky, W. P. West, J. H. Yu, J. A. Boedo, B. D. Bray, S. Brezinsek, N. H. Brooks, M. E. Fenstermacher, M. Groth, E. M.
Hollmann, A. Huber, A. W. Hyatt, S. I. Krasheninnikov, C. J. Lasnier, A. G. McLean, R. A. Moyer, A. Yu. Pigarov, V. Philipps, A. Pospieszczyk, R. D. Smirnov, J. P. Sharpe, W. M. Solomon, J. G. Watkins, and C. P. C. Wong, "Dust studies in DIII-D and TEXTOR," Nucl. Fusion 49, 085022 (2009).

${ }^{20} \mathrm{~J}$. Williams, "Application of tomographic particle image velocimetry to studies of transport in complex (dusty) plasma," Phys. Plasmas 18, 050702 (2011).

${ }^{21}$ N. Oxtoby, J. Ralph, C. Durniak, and D. Samsonov, "Tracking shocked dust: State estimation for a complex plasma during a shock wave," Phys. Plasmas 19, 013708 (2012).

${ }^{22}$ R. Gandy, S. Willis, and H. Shimoyama, "Initial experiments in the Idaho dusty plasma device," Phys. Plasmas 8, 1746 (2001).

${ }^{23}$ Y. Feng, J. Goree, and B. Liu, "Errors in particle tracking velocimetry with high-speed cameras," Rev. Sci. Instrum. 82, 053707 (2011); e-print arXiv: 1104.3540 .

${ }^{24} \mathrm{~K}$. Statsenko, "The measurement of kinetic temperature of dusty component of complex plasma in RF-discharge," AIP Conf. Proc. 799, 438-441 (2005).

${ }^{25}$ Y. Feng, J. Goree, and B. Liu, "Accurate particle position measurement from images," Rev. Sci. Instrum. 78, 053704 (2007).

${ }^{26}$ G. M. Gibson, J. Leach, S. Keen, A. J. Wright, and M. J. Padgett, "Measuring the accuracy of particle position and force in optical tweezers using high-speed video microscopy," Opt. Express 16, 14561 (2008).

${ }^{27}$ O. Otto, J. L. Gornall, G. Stober, F. Czerwinski, R. Seidel, and U. F. Keyser, "High-speed video-based tracking of optically trapped colloids," J. Opt. 13, 044011 (2011).

${ }^{28} \mathrm{P}$. L. Biancaniello and J. C. Crocker, "Line optical tweezers instrument for measuring nanoscale interactions and kinetics," Rev. Sci. Instrum. 77, 113702 (2006)

${ }^{29}$ N. Ochiai, M. I. Dragila, and J. L. Parke, "Three-dimensional tracking of colloids at the pore scale using epifluorescence microscopy," Vadose Zone J. 9, 576 (2010).

${ }^{30}$ Y. C. Tai, S. Noelle, J. M. N. T. Gray, and K. Hutter, "Shock-capturing and front-tracking methods for granular avalanches," J. Comput. Phys. 175, 269-301 (2002).

${ }^{31}$ E. I. Corwin, E. T. Hoke, H. M. Jaeger, and S. R. Nagel, "Temporal force fluctuations measured by tracking individual particles in granular materials under shear," Phys. Rev. E 77, 061308 (2008).

${ }^{32}$ J. R. Royer, D. J. Evans, L. Oyarte, Q. Guo, E. Kapit, M. E. Möbius, S. R. Waitukaitis, and H. M. Jaeger, "High-speed tracking of rupture and clustering in freely falling granular streams," Nature 459, 1110-1113 (2009).

${ }^{33}$ M. G. Clerc, P. Cordero, J. Dunstan, K. Huff, N. Mujica, D. Risso, and G. Varas, "Liquid-solid-like transition in quasi-one-dimensional driven granular media," Nat. Phys. 4, 249-254 (2008).

${ }^{34}$ T. S. Majmudar and R. P. Behringer, "Contact force measurements and stress-induced anisotropy in granular materials," Nature 435, 1079-1082 (2005)

${ }^{35} \mathrm{~B}$. Liu, J. Goree, and Y. Feng, "Non-Gaussian statistics and superdiffusion in a driven-dissipative dusty plasma," Phys. Rev. E 78, 046403 (2008).

${ }^{36}$ A. Shahzad, M.-G. He, and K. He, "Diffusion motion of two-dimensional weakly coupled complex (dusty) plasmas,” Phys. Scr. 87, 035501 (2013). 
${ }^{37} \mathrm{~T}$. Ott and M. Bonitz, "Is diffusion anomalous in two-dimensional Yukawa liquids?,” Phys. Rev. Lett. 103, 195001 (2009).

${ }^{38}$ S. Ratynskaia, C. Knapek, K. Rypdal, S. Khrapak, and G. Morfill, "Statistics of particle transport in a two-dimensional dusty plasma cluster," Phys. Plasmas 12, 022302 (2005).
${ }^{39} \mathrm{E}$. W. $\mathrm{Ng}$ and $\mathrm{M}$. Geller, "A table of integrals of the error functions," J. Res. Natl. Bureau Stand., Sect. B: Math. Sci. 73B, 1-20 (1969).

${ }^{40}$ M. Haenggi, "On distances in uniformly random networks," IEEE Trans. Inf. Theory 51, 3584-3586 (2005). 